\title{
Baryon spectroscopy with spatially improved quark sources*
}

\author{
T. Burch, C. Hagen $\dagger$, D. Hierl, and A. Schäfer \\ Institut für Theoretische Physik \\ Universität Regensburg \\ D-93040 Regensburg, Germany. \\ E-mail: christian.hagendphysik.uni-regensburg.de
}

\section{Christof Gattringer, L. Ya. Glozman, and C. B. Lang}

Institut für Physik, FB Theoretische Physik

Karl-Franzens-Universität Graz

A-8010 Graz, Austria.

\begin{abstract}
We study baryons on the lattice with a special focus on excited states. For that purpose we construct several interpolators which differ in their Dirac structure. These interpolators are built from Jacobi smeared quarks with different widths in order to allow for operators with improved spatial wavefunctions. We compute all cross correlations and use the variational method to determine which combinations of operators have best overlap with ground and excited states. Our approach yields promising results for the spin- $\frac{1}{2}$ baryons: nucleon, $\Sigma, \Xi$ and $\Lambda$. For the spin- $\frac{3}{2}$ baryons, $\Delta$ and $\Omega$, we obtain results which are consistent with results of other groups.
\end{abstract}

XXIIIrd International Symposium on Lattice Field Theory

25-30 July 2005

Trinity College, Dublin, Ireland

\footnotetext{
${ }^{*}$ For the Bern-Graz-Regensburg (BGR) Collaboration.

† Speaker.

${ }^{\ddagger}$ Supported by Fonds zur Förderung der Wissenschaftlichen Forschung in Österreich, project P16823-N08.
} 


\section{Introduction}

Ground state spectroscopy in quenched lattice calculations appears to be well understood. Excited state spectroscopy, however, is still a challenging task. There are two major problems: First, one has to improve the overlap of the interpolating fields with excited states. Excited hadron states include also radial excitations and thus nodes in their radial wavefunction. Allowing for such nodes is important and should be implemented in a lattice calculation to obtain more realistic results. The second problem is finding reliable means to disentangle ground and excited states in the hadron spectrum. Our method of choice is a variational approach [1, 2]. Some preliminary results have been presented elsewhere [3, 4, 可]. Two larger publications are in preparation.

\section{Simulation details}

We perform quenched lattice calculations using CI fermions [6, 7]. For our simulations we use two lattices with the same spatial volume of about $2.4 \mathrm{fm}$. The lattice spacings are $a=0.148 \mathrm{fm}$ and $a=0.119 \mathrm{fm}$ for our $16^{3} \times 32$ and $20^{3} \times 32$ lattices, respectively. These values are determined from the Sommer parameter [8]. We compare our results with other ways of setting the scale, namely the mass of the rho meson and the nucleon, and find that they are consistent within error bars. We are interested in baryons containing only light quarks as well as those with strange quark content. We therefore determine the mass parameter of the strange quark, using the pseudoscalar kaon mass as input.

\section{The Method}

In order to obtain reliable results for excited states it is important to have a large basis of operators which is as complete as possible. One way to increase the number of operators is to choose different Dirac structures to combine the quark spinors. Spin and parity of the hadrons pose some restrictions on that choice. Another way to increase the basis and to enhance the overlap of the interpolating fields with the physical states is to improve the spatial wavefunction of the quark sources. We use Jacobi smearing to produce Gaussian-type gauge covariant sources with two different widths, a narrow and a wide one (see Figure 1). These sources can then be combined (with a relative minus sign) to allow for a node in the radial wavefunction of a baryon interpolator. The sign factors and optimal combination are not put in by hand but chosen via the variational method, as discussed below. We then use these sources together with three different Dirac structures to obtain a basis of 24 operators.

From these interpolators we construct a matrix of correlators and then apply the variational method which has been proposed by Michael [1] and later refined by Lüscher and Wolff [2]. One has to solve a generalized eigenvalue problem. The advantage of this approach is that the system has full freedom to choose the relative contributions of the different interpolators in the diagonalization. These weighting factors can be extracted from the components of the eigenvectors. The mass is obtained by looking at the eigenvalues, which in leading order behaves as

$$
\lambda^{(k)}(t) \propto e^{-t M_{k}} .
$$




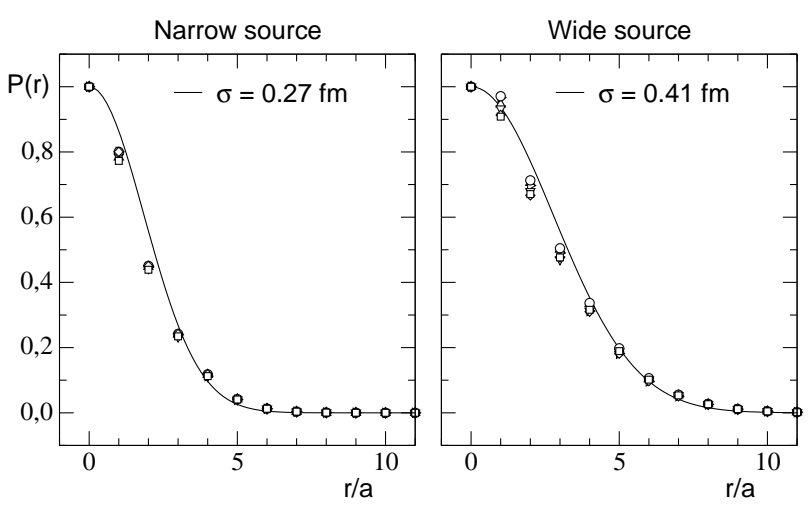

Figure 1: Profiles of the narrow and wide source. The symbols are our data points and the curves are the target distributions which we approximate by the profiles.

From these eigenvalues we then construct effective mass plots by using

$$
M_{k}^{e f f}\left(t+\frac{1}{2}\right)=\ln \left(\frac{\lambda^{(k)}(t)}{\lambda^{(k)}(t+1)}\right) .
$$

Where we find a plateau in these plots we conclude that the signal of the considered state is disentangled from higher excitations. We then fit the eigenvalue to a single exponential according to Eq. (3.1). In the following we present the results of these fits for different baryons. The application of our techniques to meson spectroscopy is presented in [9].

\section{Discussion of the Results}

In Figure 1 we show the results of our calculations for the nucleon, $\Sigma$ and $\Xi$. The behavior of these baryons is very similar. We use interpolators of similar form, except for their quark content. For the nucleon we use an interpolator with three light quarks; for $\Sigma$ and $\Xi$ we then replace one or two of the light quarks by a strange quark with fixed mass parameter. Thus we can restrict ourselves to mainly discussing the nucleon results. The same arguments then also hold for $\Sigma$ and $\Xi$, except that their dependence on the pion mass squared (and thus the light quark mass) is weaker.

For the positive parity ground state nucleon, we find results on both lattices which are consistent with each other and extrapolate very well to the value found by experiment. For the first excited state in the positive parity channel, a state which should correspond to the Roper resonance, we obtain results that are not conclusive. For the coarser lattice we cannot identify any effective mass plateaus at quark masses below $a m<0.08$. On the finer lattice there are indications for effective mass plateaus down to $a m=0.02$, but our current statistics does not allow for a reliable analysis (we omit data points with am $<0.04$ ). However, the third eigenvalue, corresponding to the second excitation, shows a considerably better signal. This might be understood from the different nature of Roper and N(1710) [3, 10]. In the negative parity channel, for both lattices we find two low-lying states which are nearly degenerate. The results on the finer lattice are lower than for the coarse lattice and extrapolate reasonably well to the experimental values for $\mathrm{N}(1535), \mathrm{N}(1650)$. For the $\Sigma$ and $\Xi$ channels we find better quality for the Roper-like states at small quark masses. 


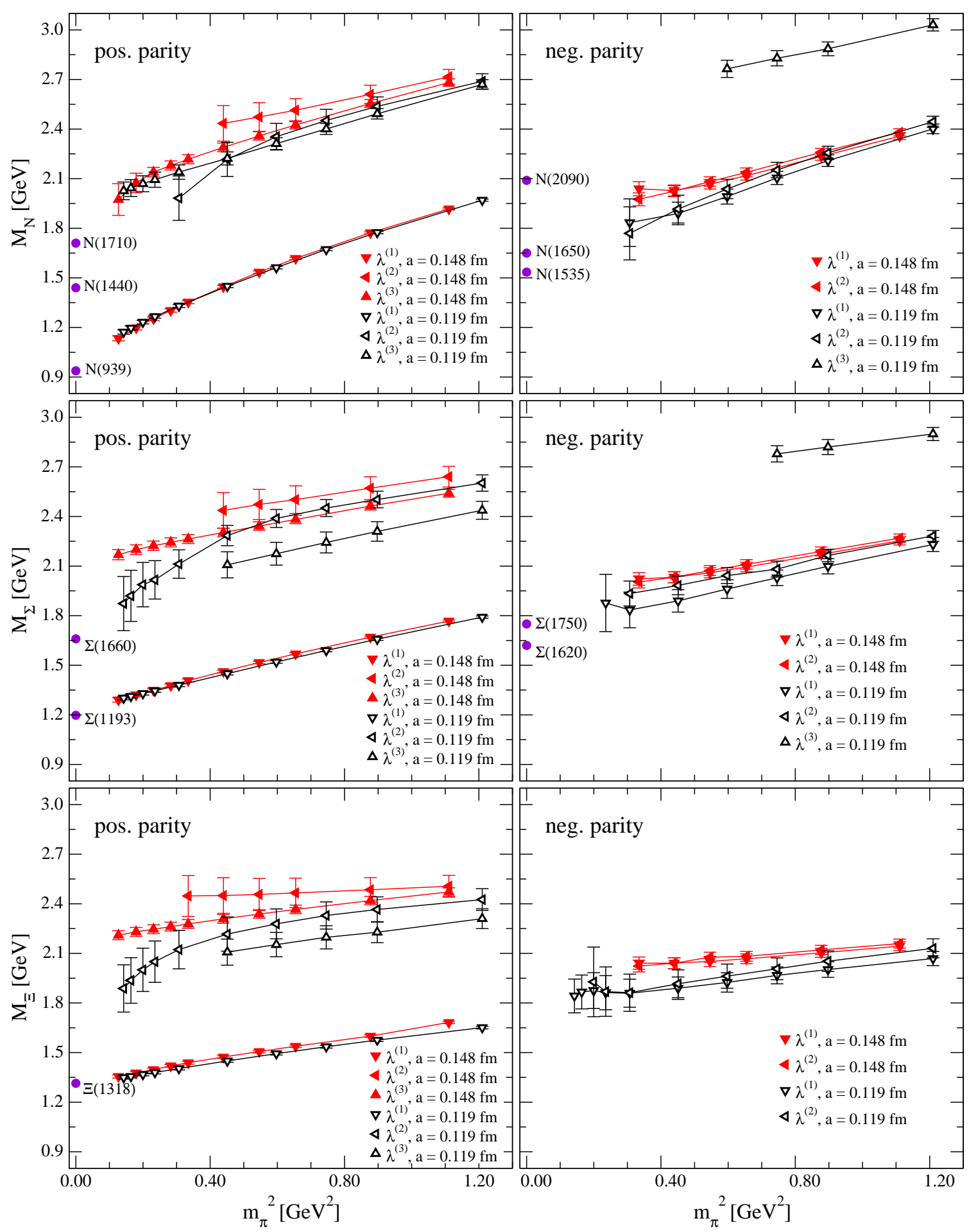

Figure 2: Results for the nucleon, $\Sigma$, and $\Xi$ (from top to bottom) versus the pion mass squared. In each case, the left-hand side plot shows the positive parity states while the right-hand side plot is for the negative parity channel. The results obtained from the $16^{3} \times 32$ lattice are represented by filled symbols while those for $20^{3} \times 32$ by open ones. 


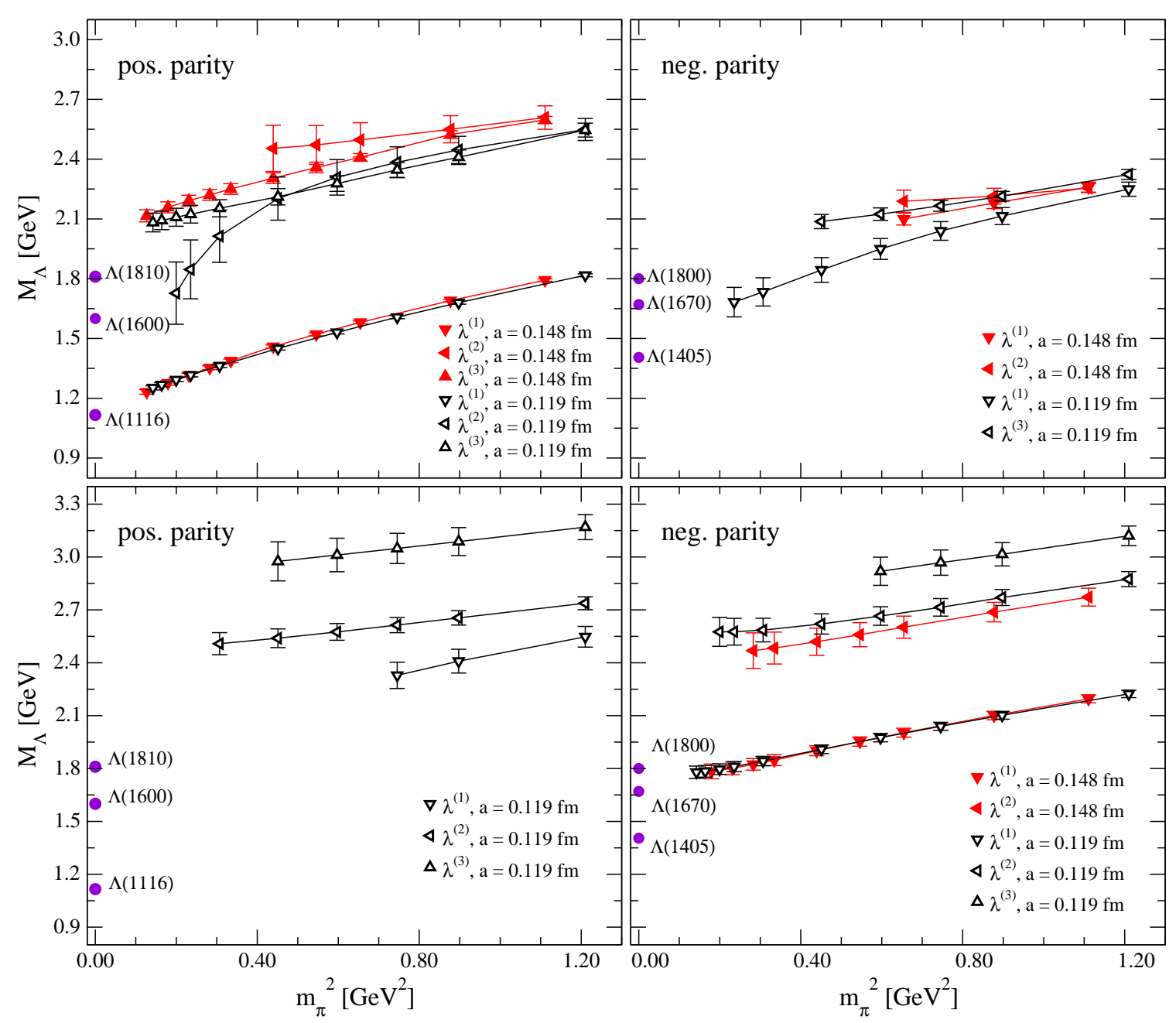

Figure 3: Results for the $\Lambda$ flavor octet interpolator (top) and the $\Lambda$ flavor singlet interpolator (bottom).

For the $\Lambda$, shown in Figure 3, we compare results from an interpolator having mainly overlap with a flavor octet and one which is a pure singlet. The octet shows a similar behavior as the other octet baryons. Again, we find good results for the positive parity ground state and a drop for the first excited state in the positive parity channel on the finer lattice.

For the flavor singlet $\Lambda$ with positive parity we find effective mass plateaus only for the finer lattice and at large quark mass. On the negative parity side, we find a strong signal for the ground state which extrapolates essentially higher than the physical $\Lambda(1405)$. This is in agreement with previous lattice calculations.

The results for the $\Delta$ are shown in Figure $\emptyset$. A naive extrapolation to the chiral limit is about $20 \%$ too high for both parities. This finding is in agreement with other lattice studies. The vertical lines in the figure indicate the point where all three quarks have the mass of the strange quark. The corresponding state is the $\Omega$ resonance and we find excellent agreement with the experimental number. This indicates that for the $\Delta$ in full QCD, chiral dynamics, which is absent in the quenched approximation, is important. 


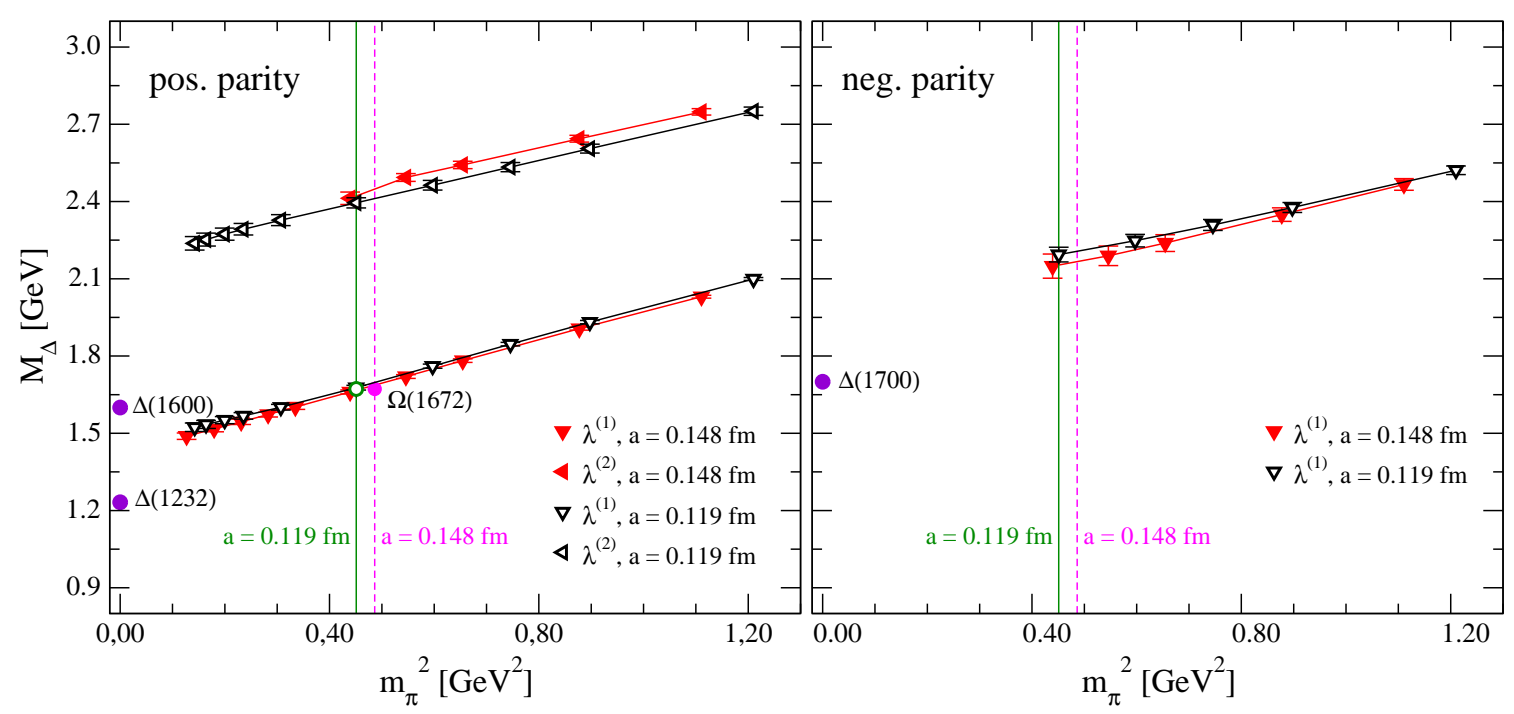

Figure 4: Results for $\Delta$ and $\Omega$. The vertical lines denote the pion masses squared corresponding to the valued of the strange quark mass on our lattices (dashed for the $16^{3} \times 32$ lattice and full for $20^{3} \times 32$ ).

\section{References}

[1] C. Michael, Adjoint sources in lattice gauge theory, Nucl. Phys. B 259 (1985) 58.

[2] M. Lüscher and U. Wolff, How to calculate the elastic scattering matrix in two-dimensional quantum field theories by numerical simulation, Nucl. Phys. B 339 (1990) 222.

[3] T. Burch, et al. [BGR (Bern-Graz-Regensburg) Collaboration], Spatially improved operators for excited hadrons on the lattice, Phys. Rev. D 70 (2004) 054502 [hep-lat / 0405006 ].

[4] T. Burch, et al. [BGR (Bern-Graz-Regensburg) Collaboration], Excited hadrons from improved interpolating fields, Nucl. Phys. Proc. Suppl. 140 (2005) 284 [hep-lat / 0409014 ].

[5] T. Burch, et al. [BGR (Bern-Graz-Regensburg) Collaboration], Masses of excited baryons from chirally improved quenched lattice QCD, Nucl. Phys. A 755 (2005) 481 [nucl-th/ 0501025 ].

[6] C. Gattringer, A new approach to Ginsparg-Wilson fermions, Phys. Rev. D 63 (2001) 114501 [hep-lat/0003005].

[7] C. Gattringer, I. Hip, and C. B. Lang, Approximate Ginsparg-Wilson fermions: A first test, Nucl. Phys. B 597 (2001) 451 [hep-lat/ 0007042 ].

[8] C. Gattringer, R. Hoffmann, and S. Schaefer, Setting the scale for the Lüscher-Weisz action, Phys. Rev. D 65 (2002) 094503 [hep-lat/ 0112024 ].

[9] T. Burch, et al. [BGR (Bern-Graz-Regensburg) Collaboration], Excited meson spectroscopy with chirally improved fermions, PoS(LAT2005)097.

[10] D. Brömmel, et al. [BGR (Bern-Graz-Regensburg) Collaboration], Excited nucleons with chirally improved fermions, Phys. Rev. D 69 (2004) 094513 [hep-ph/ 0307073 ]. 